\title{
Patterns of Bimanual Interference Reveal Movement Encoding within a Radial Egocentric Reference Frame
}

\author{
Stephan P. Swinnen ${ }^{1}$, Natalia Dounskaia ${ }^{2}$, and Jacques Duysens ${ }^{3}$
}

\begin{abstract}
Constraints on interlimb coordination have been studied intensively in past years with a primary focus on temporal features. The present study addressed spatial constraints or the degree of directional interference as a function of different line combinations between the upper limbs as well as the modulation of this interference as a result of different board orientations within the performer's workspace. This paradigm was used to address a prominent theme in motor neuroscience, namely whether (bimanual) movements are encoded within an allocentric reference frame (pattern of interference invariant with respect to extrinsic space) or
\end{abstract}

\section{INTRODUCTION}

Movements that can be produced easily with the left or right upper limb separately can become very difficult when performed together with both limbs. Such bimanual skills often require substantial amounts of practice as athletes, musicians, and other expert performers demonstrate. In past decades, an intensive research program has been initiated to reveal the nature of the constraints on interlimb coordination (Swinnen, Heuer, Massion, \& Casaer, 1994; Carson, Byblow, \& Goodman, 1993; Kelso, Southard, \& Goodman, 1979). This has resulted in a better understanding of the central nervous system's (CNS) limitations in dealing with the parallel organization and control of multiple tasks. Multiple task performance is essential for producing common daily activities and is of focal interest to cognitive neuroscience.

Previous work has identified timing as a major parameter that constrains interlimb coordination. For example, bimanual finger tapping is relatively easy when one temporal basis is an integer multiple of the other (e.g., a 1:1 or 2:1 ratio). However, when timing ratios are less compatible (e.g., a 2:3 ratio), performance is more difficult and largely depends on the capability to develop an integrated temporal structure (Summers, Rosenbaum, Burns, \& Ford, 1993; Deutsch, 1983).

\footnotetext{
${ }^{1}$ Katholieke Universiteit Leuven, Belgium, ${ }^{2}$ Arizona State University, ${ }^{3}$ Katholieke Universiteit Nijmegen, Netherlands

within an egocentric reference frame (pattern of interference invariant relative to the center of the performer's action space, i.e., intrinsic). The observed patterns of interference revealed that movements are primarily encoded within a radial egocentric reference frame in which the performer is the center of action space. The present psychophysical findings converge with primate single-cell recording studies in which the direction has been identified as a primary movement parameter that is encoded in various brain regions, thereby constituting a principal determinant of bilateral interference.

Much less attention has been devoted to the constraining role of spatial parameters, for example, amplitude and direction. When movements with different amplitudes have to be produced together by both upper limbs, assimilation effects emerge (Spijkers \& Heuer, 1995; Sherwood, 1994; Marteniuk, MacKenzie, \& Baba, 1984). Directional interactions have been studied only occasionally (Swinnen, Jardin, Meulenbroek, Dounskaia, \& Hofkens-Van Den Brandt, 1997; Swinnen et al., 1998; Franz, Zelaznik, \& McCabe, 1991), although it is clear from animal studies using single-cell recording techniques that movement direction is a primary parameter directly or indirectly encoded in various brain areas (for reviews, see Georgopoulos, 1991, 1995).

To account for patterns of contralateral interference during bimanual performance, we rely on the working hypothesis that information pertaining to movement direction is coded within the CNS and is normally exchanged between hemispheres (Donchin, Cardoso de Oliveira, \& Vaadia, 1999; Swinnen et al., 1997), resulting in interference. Since these patterns of interference are involuntary and even difficult to suppress, they are considered the spontaneous behavioral expressions of default movement encoding processes in higher order cortical regions involved in bimanual coordination. In this respect, it is of interest that split brain patients lack such interference and are able to successfully produce movements in different directions with both upper limbs simultaneously (Eliassen, Baynes, \& Gazzaniga, 1999; Franz, Eliassen, Ivry, \& Gazzaniga, 
1996). According to Eliassen et al. (1999), directional information is exchanged between cortical regions via the posterior part of the corpus callosum.

A central question is whether directional interactions emerge within the confines of an egocentric or allocentric reference frame. Single-cell recording studies on directional encoding during unilateral aiming have revealed equivocal interpretations: Whereas some have referred to movement encoding within an allocentric reference frame (Georgopoulos, 1991, 1995), others have hinted at coding within an egocentric reference frame (Scott \& Kalaska, 1995; Caminiti, Johnson, Galli, Ferraina, \& Burnod, 1991) or a combination of movement and muscle representation (Kakei, Hoffman, \& Strick, 1999).

In the present study, we addressed whether the aforementioned observations can be extrapolated to bimanual tasks in humans and whether a psychophysical approach can help to shed new light on this controversy. It is hereby assumed that patterns of directional interference during bimanual movements provide hints about the reference frame(s) within which these movements are possibly organized. Thus, although the representation of space may be an emergent property at the neural level, its existence may be reflected at the level of overt behavior in the form of a particular geometrical structure in the observed interference.

Subjects produced a bimanual cyclical line drawing task on a double digitizer setup, positioned in the horizontal plane (Figure 1). With their left limb, participants were asked to draw vertical lines repetitively (line task) while orientation in the right limb was to be varied in a clockwise manner with steps of $45^{\circ}$ (star task). More specifically, the star task started off with drawing vertical lines after which orientation was shifted with $45^{\circ}$ every time a series of five line drawings was completed within each orientation level and until a series of eight successive sequences was performed. In the present task, of particular interest was to study whether the directional shifts in the right limb affected the production of vertical line drawings in the left limb. In this respect, it was hypothesized that parallel line orientations in both limbs would generate minimal or no interference whereas orthogonal orientations would generate maximal directional interference.

Building on the aforementioned hypothesis, the next question we addressed was whether bilateral performance of parallel versus orthogonal line combinations should be understood within the confines of an egocentric or allocentric reference frame. Therefore, the position of the digitizer boards within the workspace of the performer was manipulated, either in a symmetrical or asymmetrical manner with respect to the longitudinal axis of the performer (Figure 1). This resulted in interboard angles of $0^{\circ}, 45^{\circ}$, and $90^{\circ}$. The comparison of the interference patterns observed between the $0^{\circ}$ and $90^{\circ}$ interboard angles was considered of particular interest. This is because within extrinsic space coordi- nates, parallel lines with a $0^{\circ}$ interboard angle along the $y$-axis of the digitizer would become orthogonal lines with a $90^{\circ}$ interboard angle and vice versa for the orthogonal line segment combinations. Accordingly, movement encoding within an allocentric reference frame was hypothesized to result in a shift of the pattern of interference with $90^{\circ}$ during manipulation of the digitizer boards. Conversely, from the perspective of intrinsic coordinates (egocentric reference frame), the pattern of interference was hypothesized to remain invariant across manipulations of interboard angle because the line drawings would not change their orientation relative to the longitudinal axis of the performer. More specifically, the two parallel line drawings on digitizers with a $0^{\circ}$ interboard angle are both oriented radially relative to the body and maintain their radial orientation when the interboard angle is shifted to $90^{\circ}$. Thus, the manipulation of interboard angle was assumed to reveal critical information about the reference frame within which bimanual movements are encoded.

\section{RESULTS}

Typical patterns of interference as a result of bimanual performance are illustrated in Figure 2. The example trial from a representative performer (Figure 2a) illustrates that unimanual trials are performed successfully whereas bimanual trials demonstrate pronounced difficulties, particularly with respect to the left line drawing task. More specifically, the vertical orientation cannot be maintained in the left limb when a nonvertical component of the star drawing is performed with the right limb.

This is further illustrated with the group data of variability $(S D)$ of orientation during left line (Figure $2 \mathrm{~b}$, left) and right star drawing (Figure 2b, right) under uni- and bimanual performance conditions that will receive primary attention. It is evident that the bimanual drawing is much more variable than the unimanual one, and this effect is more pronounced for the left line task than for the right star task (see significant performance condition effect for $S D$ of line and star drawing in Table 1). The performance condition effect also interacts significantly with sequence. For the star, the most stable condition is always the straight-ahead orientation (12 o' clock), while the least stable condition is the bimanual task in which the right hand has to perform a movement that is orthogonal to the left line drawing (i.e., at the 3 and 9 o' clock orientations, Figure 2b, right side). This orthogonal condition is also clearly the most unstable one for the left line drawing (see 3 and 9 o'clock positions in Figure $2 \mathrm{~b}$, left side), introducing a significant bias away from the vertical target orientation of $90^{\circ}$ and giving rise to a high degree of variability in orientation. More specifically, mean orientation angles (not shown) for the left limb during bimanual performance across the eight sequences of the star movement and starting from the 


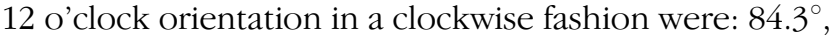
$77.5^{\circ}, 68.7^{\circ}, 76.5^{\circ}, 84.9^{\circ}, 73.1^{\circ}, 63.4^{\circ}$, and $74.2^{\circ}$.

The aforementioned findings resulted in a significant effect for sequence as well as a Sequence $\times$ Performance Condition interaction with respect to the $S D$ scores of the line and star drawing as well as with respect to the mean orientation scores for line drawing (Table 1). Hence, both the findings with respect to the line and star drawing tasks suggest that orientation interference is minimal or even absent during the bimanual performance of parallel lines and maximal during the performance of orthogonal lines.

To obtain insights into the reference frames within which bimanual movements are organized, subjects were to perform these bimanual movements in different parts of the workspace (Figure 1). Under the assumption of an egocentric reference frame, the pattern of interference is predicted to be the same across the three board orientations. Conversely, evidence for an allocentric reference frame would become evident as a shift in the pattern of interference with $45^{\circ}$ and $90^{\circ}$ (relative to the parallel board orientation) during performance with $45^{\circ}$ and $90^{\circ}$ interboard angles, respectively. Data for the $0^{\circ}$ and $90^{\circ}$ interboard angles are shown in Figure $3 \mathrm{a}$ and exemplify a very similar pattern of interference across both symmetrical board orientations. The data for the $45^{\circ}$ interboard angle (not shown here) was positioned in between. This implies that the pattern of interference is invariant with respect to an egocentric but not with respect to an allocentric reference frame. Indeed, the most stable pattern during the parallel board orientation is observed when the lines are parallel in allocentric space and the least stable when the lines are orthogonal. Conversely, when the boards are tilted $90^{\circ}$, the most stable pattern emerges when the lines are orthogonal in allocentric space and the least stable when the lines are parallel. Accordingly, accounting for the data within an egocentric reference frame is more parsimonious. Nevertheless, a small contribution from coding within an allocentric frame may have been present, since the variability at the 3 and 9 o'clock orientations was significantly lower in the $90^{\circ}$-tilted (Figure 3a, gray line) than in the $0^{\circ}$ interboard angle condition (Figure $3 \mathrm{a}$, black line). It is noteworthy to add here that this former movement combination was associated with drawing parallel lines in extrinsic space. This effect was supported by the significant Board Orientation $\times$ Sequence as well as the Performance Condition $\times$ Board Orientation $\times$ Sequence interaction for the $S D$ scores of the left line orientations (Table 1 , left side).

It may be argued that the stable performance of bimanual movements along radial directions with respect to the body midline is not so much a result of the egocentric reference frame but merely a consequence of the symmetrical activation patterns in the arm muscles concerned. To examine this symmetry argument, the experiments were repeated under asymmetric conditions (Figure 1, Experiment 2). Whereas bilateral shoulder angles at starting position were the same in the previous experiment as a result of the symmetrical board orientations, different angles were obtained in the "asymmetric experiment" by means of rotation of the right digitizer board with $45^{\circ}$ and $90^{\circ}$, relative to the left board.

The findings of this experiment were largely consistent with those of the previous experiment and exemplify the evidence for predominant encoding within an egocentric reference frame (see Table 1, right portion). The highest $S D$ scores in the left limb can be observed at the 3 and 9 o'clock orientations (Figure 3b) and this is associated with the largest absolute deviations from the mean target orientation of $90^{\circ}$ (not shown). These observations were supported by a significant Performance Condition $\times$ Sequence interaction for the mean and $S D$ of left line orientation (Table 1 , right side).
Figure 1. Schematic illustration of the different task combinations in Experiments 1 and 2. In Experiment 1 (top), the interboard angles are $0^{\circ}, 45^{\circ}$, or $90^{\circ}$, while the left and right shoulder positions are symmetrical. Similar interboard angles are used in Experiment 2 (bottom) but with asymmetrical shoulder positions.

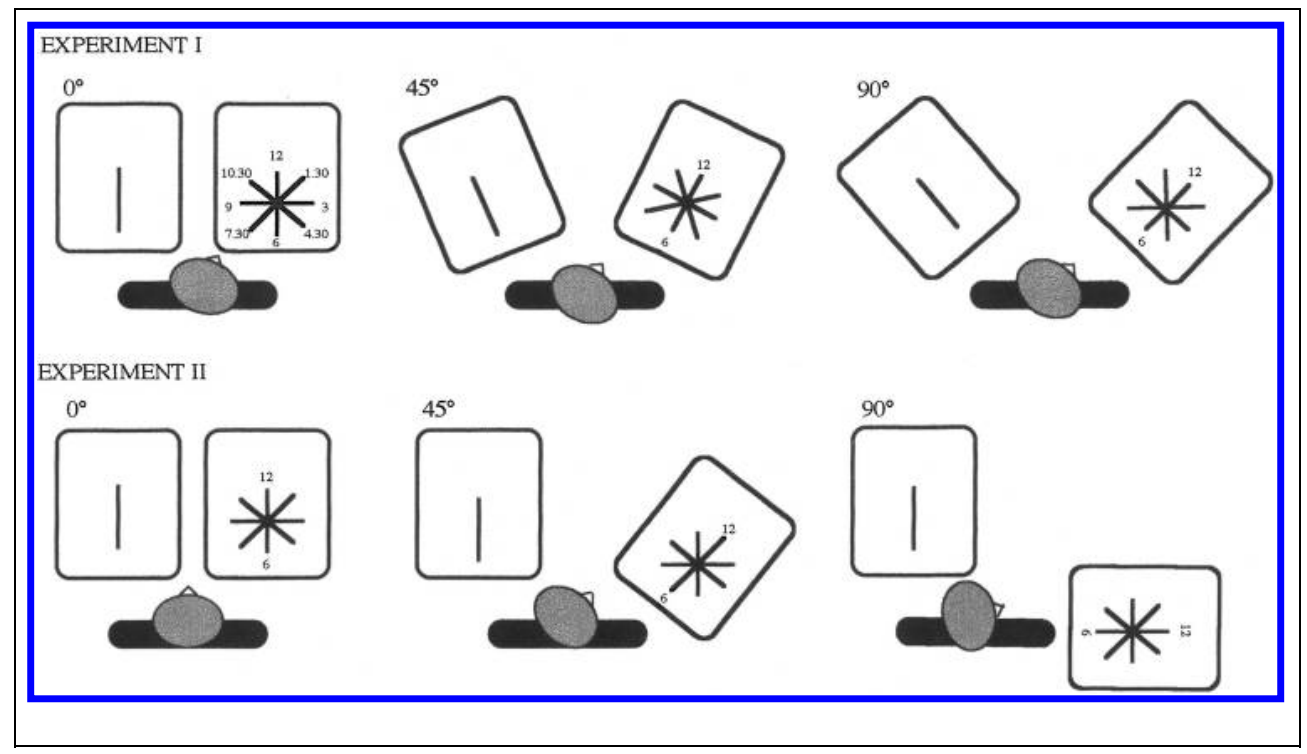




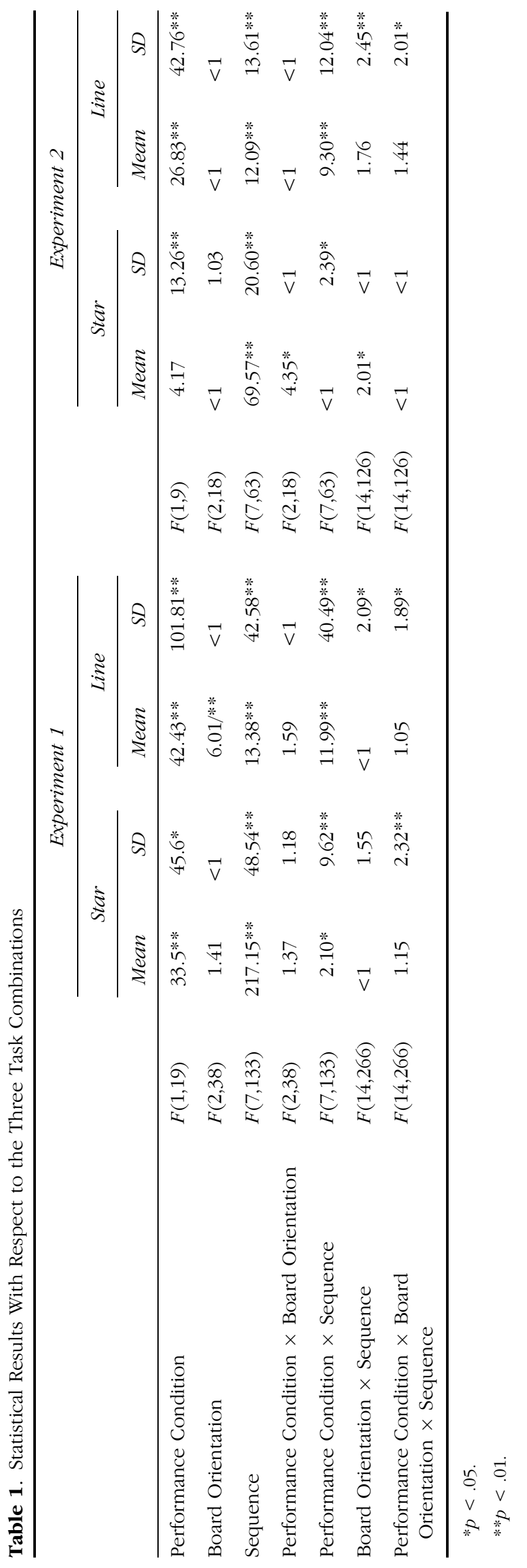


Figure 2. Example of kinematics for the left line and right star drawings (a) and polar plots representing $S D$ of orientation for the left line and right star drawings during unimanual and bimanual task conditions

(b). An example of a unimanual and bimanual trial is shown in

(a) for a representative subject. Interference is particularly evident in the left limb during bimanual performance. In (b), group results with respect to the $S D$ of orientation during unimanual and bimanual performance is presented for the left line and right star drawings. The angle in the polar plots (b) refers to the eight orientations that are sequentially generated during star drawing. The radius represents the $S D$ of orientation angle that was actually obtained during each of these eight sequences. The $S D$ of orientation angle for the sequential epochs of left line drawing is also plotted against the eight different clock positions of the star drawing to better visualize the pattern of interference over the course of the trial. Unimanual (black squares) and bimanual (gray circles) results are plotted together to illustrate the changes in orientation $S D$ as a result of simultaneous performance. The larger the $S D$

scores, the higher the directional variability. It is generally evident from both plots that the $S D$ scores are higher during bimanual than during unimanual performance. The $S D$ scores are specifically elevated when the directional drawing requirements differ between the star and line drawing. The $S D$ scores are highest at the 3 and 9 o'clock orientations and adjacent ones and lowest at the 12 and 6 o'clock orientations.
Nevertheless, the differences in variability under tilted board conditions were somewhat reduced as compared to the parallel board condition at the 3 and 9 o'clock orientations, perhaps indicating an increasing effect of allocentric coding (see significant Board Orientation $\times$ Sequence and Performance Condition $\times$ Board Orientation $\times$ Sequence interaction, Table 1 , right side). In general however, the lack of symmetry with respect to the body has little effect on the dominant pattern in the observed variability scores, making it unlikely that the basic observations from the previous experiment were merely based on symmetry in muscle activation.

\section{DISCUSSION}

The behavioral interpretation of the present findings is that the interference between both upper limbs is minimal when lines are drawn simultaneously along radial orientations that diverge from the center of action space, that is, the longitudinal axis of the body or its extension to the shoulder. Maximal interference is ob- served when a radial and orthogonal-to-radial orientation has to be generated simultaneously, irrespective of the location of these movements within the workspace of the performer. At first sight, this egocentric reference frame is a concomitant of the general preference to produce symmetrical or mirror-image limb movements that constitutes one of the most archaic modes of interlimb coordination (Swinnen et al., 1997; Semjen, Summers, \& Cattaert, 1995; Stucchi \& Viviani, 1993) that is largely unaffected by brain pathology (Wiesendanger, Wicki, \& Rouiller, 1994). However, it is not a mere consequence of the preference for homologous muscle activation because the results with asymmetric positioning of the digitizers were largely the same as for the symmetric condition. In addition, bilateral kinematic interference remains largely the same when the underlying patterns of muscle activation for producing the drawings are dramatically changed by spring loading under invariant kinematics (Swinnen, Dounskaia, Levin, \& Duysens, 2001). This is rather remarkable as spring loading of the arm producing the star drawing resulted 
Figure 3. $S D$ of orientation angle of the left line drawing during the $0^{\circ}$ - and $90^{\circ}$-tilted board orientations with symmetrical (a) and asymmetrical (b) shoulder angles. Group results for the $0^{\circ}$ interboard angle are presented in black and for the $90^{\circ}$ interboard angle in gray. With respect to both the symmetric (a) and asymmetric (b) shoulder positions, the pattern of interference observed during the $0^{\circ}$ - and $90^{\circ}$-board orientations is compatible with an egocentric reference frame and not with an allocentric reference frame because the pattern of interference is largely invariant under the former assumption. As can be observed, the overall pattern of interference is similar during parallel and $90^{\circ}$-tilted conditions.

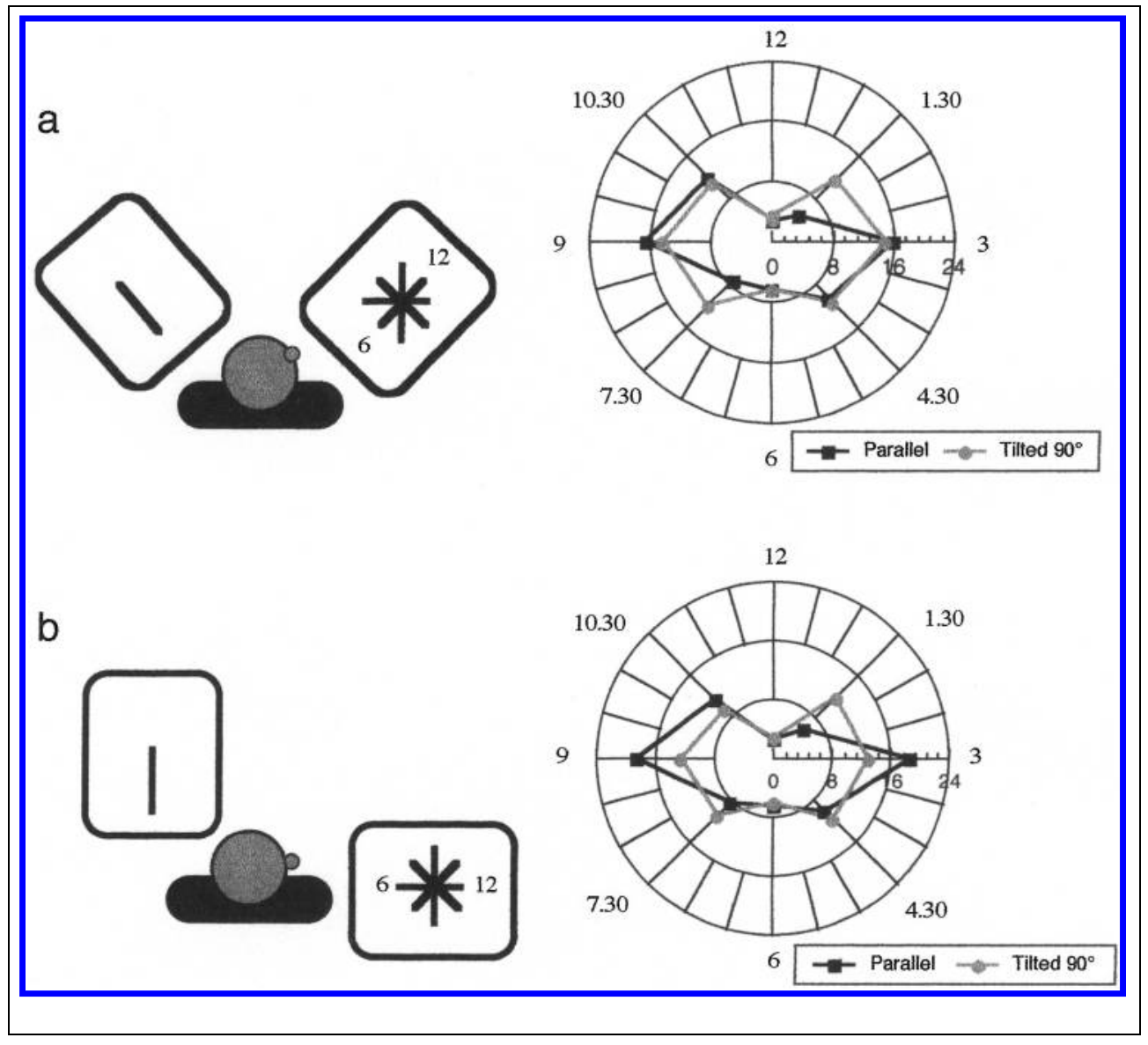

in considerable changes in the amount and ratio of activity of the elbow and shoulder muscles as well as the pattern of relationships between agonists and antagonists (Levin \& Swinnen, submitted). Yet, the orientation interference between the left and right limb was unaffected by these modulations in muscle activity and was largely determined by kinematic compatibility. This suggests that the higher cortical regions encode movement direction in a rather abstract fashion, removed from the specific patterns of muscle activation. Recent additional studies have shown the robustness of the interference effect in the face of various experimental manipulations. For example, the observed pattern of interference is equally evident when the task is performed under blindfolded conditions as opposed to visual control of the dominant limb with different head orientations, suggesting that vision and line of sight are not critical factors (Swinnen et al., unpublished data). Finally, experiments have also demonstrated that a similar pattern of interference is observed (even though reversed) when task allocation is exchanged between the dominant and nondominant limb, namely, when the line drawing task is assigned to the dominant limb and the star drawing to the nondominant limb. ${ }^{1}$ All together, the present findings provide converging evidence for the existence of an egocentric reference frame that goes beyond muscle homology. Although the present find- ings were generated during the production of bimanual movements, we entertain the hypothesis that intrinsic reference coding may also apply to other types of tasks. Accordingly, successful reaching to objects in the workspace may only require exchange of information between brain areas involved in movement planning (intrinsic reference frame) and those involved in representation of space based on visual input (extrinsic reference frame) rather than requiring complex transformations from one reference frame to the other.

In addition to the basic operation of an egocentric reference frame, subtle indications were observed for what may perhaps be interpreted as encoding within an allocentric reference frame. The $S D$ scores were slightly depressed with respect to the 3 and 9 o'clock positions during the $90^{\circ}$ board orientation, relative to the parallel board orientation, both in the symmetric and the asymmetric conditions. It is noteworthy here that the reduction in interference at these latter clock orientations was associated with the generation of parallel movements in extrinsic space.

Even though the study of reference frames has previously not been tackled by means of a bimanual task paradigm, the present approach is promising because the observed patterns of kinematic interference are "involuntary" or spontaneous behavioral expressions of natural patterns of contralateral irradiation 
of brain activity. Accordingly, the present psychophysical approach may provide an interesting window into the basic mechanisms of space representation in the human CNS.

In summary, the present findings are consistent with considerable psychophysical and physiological evidence that direction is a primary movement parameter, encoded in various brain structures (Georgopoulos, 1991, 1995). However, this coding is hypothesized to occur within the confines of an idiosyncratic radial reference frame in which the mover occupies the center of action space. Other reference frames may coexist with the current one, depending on the particular task requirements that the performer is confronted with.

\section{METHODS}

\section{Subjects}

Twenty right-handed (by self-report) participants were involved in Experiment 1 (mean age $=21$ years) and 10 in Experiment 2 (mean age $=20.5$ years). The experimental protocol was approved by the Ethical Committee of Biomedical Sciences at the Katholieke Universiteit Leuven. Informed, consent was obtained from all participants and subjects' rights were protected.

\section{Apparatus and Task}

The apparatus consisted of two $x y$-digitizing tables (LC20-TDS Terminal Display Systems) positioned in the horizontal plane (accuracy $=0.25 \mathrm{~mm}$, sampling frequency $=150 \mathrm{~Hz}$ ). Subjects held a stylus in each hand. A computer-controlled electronic metronome indicated the cycling frequency (126 beats per minute). Subjects were to draw a complete line (peak-to-peak length $=20 \mathrm{~cm}$ ) back and forth with each pulse of the metronome in $476 \mathrm{msec}$. The total duration of each trial was $21 \mathrm{sec}$. Vertical lines were performed with the left limb (line task). The right limb started off with drawing vertical lines but shifted orientation with $45^{\circ}$ in a clockwise fashion, each time a series of five lines was completed within each orientation level, and in response to the accent (higher tone) provided by the metronome beat (star task) (Figure 1, upper left drawing). Thus, the metronome paced the timing of each line drawing as well as the regular shifts in orientation. For convenience, the different orientations will be associated with the small pointer (hour hand) of a clock. Accordingly, the following clock orientations were visited: 12, 1:30, 3, 4:30, 6, 7:30, 9, and 10:30 o'clock. This implies that participants moved back and forth between opposite clock positions, thereby passing through the center of the clock during each movement cycle, e.g., from 12 to 6 o'clock and back until five full cycles were completed, from 1:30 to 7:30 o'clock and back, and so forth. Thus, line orientations were pairwise parallel with respect to the opposite clock positions. In contrast, participants moved with their left hand between the 12 and 6 o'clock positions and tried to maintain this vertical orientation across the whole duration of the trial. A template of the patterns was drawn in black on each digitizer. Participants always started at the center position of the template at initiation of each trial. To reveal the spontaneous patterns of interference, sufficient time pressure was imposed on the participant to reduce the exploitation of compensatory mechanisms.

\section{Procedure}

The line and star tasks were first performed separately during unilateral trials (unimanual), prior to their combined performance (bimanual). Two practice trials preceded each of the two unilateral test trials. No prior practice was allowed on the bimanual trials because we wanted to assess the spontaneous directional patterns of interference in the absence of training. During the bimanual trials, participants were instructed to visually monitor the star drawing task whereas the line drawing task was prohibited from vision at all times (e.g., also during unilateral performance conditions). In addition, they were to comply strictly with the temporal constraints of the metronome. These instructions were intended to maximize successful performance on the star drawing task while predominantly gating the pattern of interference towards the line drawing task. This mode of task allocation also conforms with the natural preference to allocate the more difficult of the two tasks to the dominant limb.

To investigate whether the pattern of interference was modulated as a function of arm position in space, the digitizers were oriented differently with respect to the body, either symmetrical (Experiment 1) or asymmetrical (Experiment 2) and with different interboard angles, that is, $0^{\circ}, 45^{\circ}$, and $90^{\circ}$ (Figure $1 \mathrm{a}$ and $\mathrm{b}$ ). Rotation of the trunk was prohibited to ensure that subjects could not reduce the imposed asymmetries in limb positioning.

\section{Data Analysis}

The mean angle of orientation of each line drawing with respect to the horizontal reference position $\left(=0^{\circ}\right)$ was assessed as well as its standard deviation, using statistics for directional data (Mardia, 1972). This was the primary variable of interest. The vertical orientation conformed to $90^{\circ}$. Accordingly, the scores were constrained between $0^{\circ}$ and $90^{\circ}$. Following the determination of the peak positions of each line, the ratio between $\left(y_{2}-y_{1}\right)$ and $\left(x_{2}-x_{1}\right)$ was computed. The orientation angle of each line was obtained by determining the arctan of this ratio. Accordingly, target orientation scores were $0^{\circ}, 45^{\circ}$, and $90^{\circ}$ for the lines composing the right star drawing and $90^{\circ}$ for the vertical lines composing the left line 
drawing. Separate statistical analyses were conducted on the line and star drawing results. The data of Experiments 1 and 2 were analyzed by means of $2 \times 3 \times 8$ (Performance Condition $\times$ Board Orientation $\times$ Sequence) ANOVAs. Performance condition referred to unilateral and bilateral performance, board orientation referred to the $0^{\circ}, 45^{\circ}$, and $90^{\circ}$ interboard angles, and sequence referred to the eight epochs in which direction was to be shifted during star drawing whereas the vertical line orientation was to be maintained in the left limb.

The notion of egocentric and allocentric reference frame coding leads to the following predictions. If movements are organized within an egocentric reference frame in which the performer forms the center of action space, it is hypothesized that the pattern of interference remains the same, relative to this central longitudinal axis (or its extension to the shoulder). As kinematic registrations were made on digitizers that were rotated across the workspace, this was hypothesized to result in an invariant pattern of interference across the three board orientations. If, however, movements would be organized within an allocentric reference frame, the pattern of interference was hypothesized to remain the same in extrinsic space coordinates, irrespective of the board orientations. In view of the aforementioned board manipulations, this was hypothesized to cause a shift in the pattern of interference with $45^{\circ}$ and $90^{\circ}$ for the $45^{\circ}$ and $90^{\circ}$ rotated board conditions, respectively.

\section{Acknowledgments}

Support for the present study was provided through a grant from the Research Council of the Katholieke Universiteit Leuven, Belgium (Contract No. OT/99/39) and the National Fund for Scientific Research in Belgium (Project G.0285.98). N. D. and J. D. were supported by a fellowship from the Research Council of the Katholieke Universiteit Leuven (Contract No. F/95/51 and F/99/34, respectively).

Reprint requests should be sent to Stephan P. Swinnen, Motor Control Laboratory, Department of Kinesiology, Katholieke Universiteit Leuven, Tervuurse Vest 101, 3001 Leuven, Belgium, or via e-mail: Stephan.Swinnen@FLOK.KULEUVEN.AC.BE

\section{Note}

1. When the vertical line drawing was performed with the left limb and the star drawing with the right limb, the following $S D$ scores for orientation were obtained for each of the eight sequences in the left limb: $4.06^{\circ}, 8.28^{\circ}, 20.44^{\circ}$, $12.12^{\circ}, 5.58^{\circ}, 6.17^{\circ}, 20.84^{\circ}$, and $14.76^{\circ}$ for the $12: 00,1: 30,3: 00$, $4: 30,6: 00,7: 30,9: 00$, and 10:30 o'clock orientations, respectively. When the vertical line drawing was performed by the right limb and the star drawing by the left limb, the following $S D$ scores were obtained in the right limb: $2.29^{\circ}$, $6.7^{\circ}, 19^{\circ}, 7.9^{\circ}, 4.8^{\circ}, 10.4^{\circ}, 18.7^{\circ}$, and $7.4^{\circ}$. Thus, while the $S D$ scores tended to be slightly smaller when the vertical line drawing was performed by the right (dominant) as compared to the left limb (nondominant), the observed pattern of interference was essentially preserved, namely, higher variability in the orientation of the vertical line drawing was obtained when orthogonal lines were to be drawn simultaneously as compared to parallel lines.

\section{REFERENCES}

Caminiti, R., Johnson, P. B., Galli, C., Ferraina, S., \& Burnod, Y. (1991). Making arm movements within different parts in space: The premotor and motor cortical representation of a coordinate system for reaching to visual targets. Journal of Neuroscience, 11, 1182-1197.

Carson, R. G., Byblow, W. D., \& Goodman, D. (1993). The dynamical substructure of bimanual coordination. In S. P. Swinnen, H. Heuer, J. Massion, \& P. Casaer (Eds.), Interlimb coordination: Neural, dynamical, and cognitive constraints (pp. 319-337). San Diego, CA: Academic Press.

Deutsch, D. (1983). The generation of two isochronous sequences in parallel. Perception and Psychophysics, 34, 331-337.

Donchin, O., Cardoso de Oliveira, S., \& Vaadia, E. (1999). Who tells one hand what the other is doing: The neurophysiology of bimanual movements. Neuron, 23, 15-18.

Eliassen, J. C., Baynes, K., \& Gazzaniga, M. S. (1999). Direction information coordinated via the posterior third of the corpus callosum during bimanual movements. Experimental Brain Research, 128, 573-577.

Franz, E. A., Eliassen, J. C., Ivry, R. B., \& Gazzaniga, M. S. (1996). Dissociation of spatial and temporal coupling in the bimanual movements of callosotomy patients. Psychological Science, 7, 306-310.

Franz, E. A., Zelaznik, H. N., \& McCabe, G. (1991). Spatial topological constraints in a bimanual task. Acta Psvchologica, 77, 137-151.

Georgopoulos, A. P. (1991). Higher order motor control. Annual Review of Neuroscience, 14, 361-377.

Georgopoulos, A. P. (1995). Current issues in directional motor control. Trends in Neurosciences, 18, 506-510.

Kakei, S., Hoffman, D. S., \& Strick, P. L. (1999). Muscle and movement representations in the primary motor cortex. Science, 285, 2136-2139.

Kelso, J. A. S., Southard, D. L., \& Goodman, D. (1979). On the nature of human interlimb coordination. Science, 203, 1029-1031.

Levin, O., \& Swinnen, S. P. (submitted). The effects of spring loading on unimanual and bimanual performance.

Mardia, K. V. (1972). Statistics of directional data. London: Academic Press.

Marteniuk, R. G., MacKenzie, C. L., \& Baba, D. M. (1984). Bimanual movement control: Information processing and interaction effects. Quarterly Journal of Experimental Psychology, 36A, 335-365.

Scott, S. H., \& Kalaska, J. F. (1995). Changes in motor cortex activity during reaching movements with similar hand paths but different arm postures. Journal of Neurophysiology, 73, 2563-2567.

Semjen, A., Summers, J. J., \& Cattaert, D. (1995). Hand coordination in bimanual circle drawing. Journal of Experimental Psychology, Human Perception and Performance, 21, 1139-1157.

Sherwood, D. E. (1994). Hand preference, practice order, and spatial assimilations in rapid bimanual movement. Journal of Motor Behavior, 26, 123-134.

Spijkers, W., \& Heuer, H. (1995). Structural constraints on the performance of symmetrical bimanual movements with different amplitudes. Quarterly Journal of Experimental Psychology, 48A, 716-740.

Stucchi, N., \& Viviani P. (1993). Cerebral dominance and 
asynchrony between bimanual two-dimensional movements. Journal of Experimental Psychology, Human Perception and Performance, 19, 1200-1220.

Summers, J. J., Rosenbaum, D. A., Burns, B. D., \& Ford, S. K. (1993). Production of polyrhythms. Journal of Experimental Psychology, Human Perception and Performance, 19, 416-428.

Swinnen, S. P., Dounskaia, N., Levin, O., \& Duysens, J. (2001). Constraints during bimanual coordination: The role of direction in relation to amplitude and force requirements. Behavioural Brain Research, 123, 201-218.

Swinnen, S. P., Heuer, H., Massion, J., \& Casaer, P. (Eds.) (1994). Interlimb coordination: Neural, dynamical, and cognitive constraints. San Diego, CA: Academic Press.

Swinnen, S. P., Jardin, K., Meulenbroek, R., Dounskaia, N., \&
Hofkens-Van Den Brandt, M. (1997). Egocentric and allocentric constraints in the expression of patterns of interlimb coordination. Journal of Cognitive Neuroscience, 9, 348-377.

Swinnen, S. P., Jardin, K., Meulenbroek, R., Franz, L., Dounskaia, N., \& Walter, C. B. (1998). Exploring interlimb constraints during bimanual graphic performance: Effects of muscle grouping and direction. Behavioural Brain Research, 90, 79-87.

Wiesendanger, M., Wicki, U., \& Rouiller, E. (1994). Are there unifying structures in the brain responsible for interlimb coordination? In S. P. Swinnen, H. Heuer, J. Massion, \& P. Casaer (Eds.), Interlimb coordination: Neural, dynamical, and cognitive constraints (pp. 179-207). San Diego, CA: Academic Press. 


\section{This article has been cited by:}

1. Deanna M. Kennedy, Jason B. Boyle, Chaoyi Wang, Charles H. Shea. 2014. Bimanual force control: cooperation and interference?. Psychological Research. [CrossRef]

2. Deanna M. Kennedy, Jason B. Boyle, Joohyun Rhee, Charles H. Shea. 2014. Rhythmical bimanual force production: homologous and non-homologous muscles. Experimental Brain Research . [CrossRef]

3. J. Gooijers, S.P. Swinnen. 2014. Interactions between brain structure and behavior: The corpus callosum and bimanual coordination. Neuroscience \& Biobehavioral Reviews 43, 1-19. [CrossRef]

4. Francesca Garbarini, Federico D'Agata, Alessandro Piedimonte, Katiuscia Sacco, Marco Rabuffetti, Fred Tam, Franco Cauda, Lorenzo Pia, Giuliano Geminiani, Sergio Duca, Simon J. Graham, Anna Berti. 2014. Drawing lines while imagining circles: Neural basis of the bimanual coupling effect during motor execution and motor imagery. NeuroImage 88, 100-112. [CrossRef]

5. Tetsuro Muraoka, Yuki Ishida, Takashi Obu, Larry Crawshaw, Kazuyuki Kanosue. 2013. Ipsilateral wrist-ankle movements in the sagittal plane encoded in extrinsic reference frame. Neuroscience Research 75, 289-294. [CrossRef]

6. Jolien Gooijers, Karen Caeyenberghs, Helene M. Sisti, Monique Geurts, Marcus H. Heitger, Alexander Leemans, Stephan P. Swinnen. 2013. Diffusion tensor imaging metrics of the corpus callosum in relation to bimanual coordination: Effect of task complexity and sensory feedback. Human Brain Mapping 34:10.1002/hbm.v34.1, 241-252. [CrossRef]

7. James N. Ingram, Daniel M. WolpertNaturalistic approaches to sensorimotor control 3-29. [CrossRef]

8. Nicole Wenderoth, Matthias WeigeltVisual cues influence motor coordination: behavioral results and potential neural mechanisms mediating perception-action coupling and response selection 179-188. [CrossRef]

9. R.R. Walsh, S.L. Small, E.E. Chen, A. Solodkin. 2008. Network activation during bimanual movements in humans. NeuroImage 43, 540-553. [CrossRef]

10. Julie Duque, Nagako Murase, Pablo Celnik, Friedhelm Hummel, Michelle Harris-Love, Riccardo Mazzocchio, Etienne Olivier, Leonardo G. Cohen. 2007. Intermanual Differences in Movement-related Interhemispheric Inhibition. Journal of Cognitive Neuroscience 19:2, 204-213. [Abstract] [PDF] [PDF Plus]

11. Herbert Heuer. 2006. Multiple frames of reference for bimanual co-ordination. Experimental Brain Research 175, 485-498. [CrossRef]

12. R. L. J. Meesen, N. Wenderoth, J. J. Temprado, J. J. Summers, S. P. Swinnen. 2006. The coalition of constraints during coordination of the ipsilateral and heterolateral limbs. Experimental Brain Research 174, 367-375. [CrossRef]

13. Nicole Wenderoth, Ivan Toni, Sigrid Bedeleem, Filiep Debaere, Stephan P. Swinnen. 2006. Information processing in human parieto-frontal circuits during goal-directed bimanual movements. NeuroImage 31, 264-278. [CrossRef]

14. Herbert Heuer, Wolfhard Klein. 2006. The modulation of intermanual interactions during the specification of the directions of bimanual movements. Experimental Brain Research 169, 162-181. [CrossRef]

15. Nicole Wenderoth, Filiep Debaere, Stefan Sunaert, Stephan P. Swinnen. 2005. Spatial interference during bimanual coordination: Differential brain networks associated with control of movement amplitude and direction. Human Brain Mapping 26:10.1002/ hbm.v26:4, 286-300. [CrossRef]

16. Nicole Wenderoth, Filiep Debaere, Stefan Sunaert, Stephan P. Swinnen. 2005. The role of anterior cingulate cortex and precuneus in the coordination of motor behaviour. European Journal of Neuroscience 22:10.1111/ejn.2005.22.issue-1, 235-246. [CrossRef]

17. Timothy N. Welsh, Quincy J. Almeida, Timothy D. Lee. 2005. The effect of postural stability and spatial orientation of the upper limbs on interlimb coordination. Experimental Brain Research 161, 265-275. [CrossRef]

18. Sophie Vangheluwe, Veerle Puttemans, Nicole Wenderoth, Marc Van Baelen, Stephan P. Swinnen. 2004. Inter- and intralimb transfer of a bimanual task: generalisability of limb dissociation. Behavioural Brain Research 154, 535-547. [CrossRef]

19. Jorn Diedrichsen, Rohit Nambisan, Steve W. Kennerley, Richard B. Ivry. 2004. Independent on-line control of the two hands during bimanual reaching. European Journal of Neuroscience 19:10.1111/ejn.2004.19.issue-6, 1643-1652. [CrossRef]

20. Nicole Wenderoth, Veerle Puttemans, Sophie Vangheluwe, Stephan Swinnen. 2003. Bimanual Training Reduces Spatial Interference. Journal of Motor Behavior 35, 296-308. [CrossRef]

21. S Swinnen. 2003. Directional interference during bimanual coordination: is interlimb coupling mediated by afferent or efferent processes. Behavioural Brain Research 139, 177-195. [CrossRef]

22. Stephan P. Swinnen. 2002. INTERMANUAL COORDINATION: FROM BEHAVIOURAL PRINCIPLES TO NEURALNETWORK INTERACTIONS. Nature Reviews Neuroscience 3, 348-359. [CrossRef] 